\title{
Iontophoretic Fraction Collection for Coupling Capillary Zone Electrophoresis with Matrix-Assisted Laser Desorption/lonization Mass Spectrometry
}

\author{
Jean-Marc Busnel, Jacques Josserand, Niels Lion, and Hubert H. Girault* \\ Laboratoire d'Electrochimie Physique et Analytique, Station 6, Ecole Polytechnique Fédérale de Lausanne, \\ $\mathrm{CH}-1015$, Lausanne, Switzerland
}

An automated fraction collection interface has been developed for coupling capillary electrophoresis (CE) with matrix-assisted laser desorption/ionization mass spectrometry (MALDI MS). This fraction collection approach is based on electromigration and diffusion and does not rely on the presence of a liquid junction, sheath-liquid, electro-osmotic flow, or a superimposed hydrodynamic flow. Neutrally coated capillary with negligible electroosmosis can thus be used to provide high-efficiency separations of biological compounds. The in-capillary separation resolution is totally independent from the spotting process. CE-separated species can be collected either in a multiwell plate or directly on a MALDI target. In the present work, an eight-protein mixture, submitted to trypsin proteolysis, has been used as a sample test and separations have been conducted in $50 \mu \mathrm{m}$ i.d. neutrally coated capillaries. As compared to direct MALDI MS analysis, the integration of $\mathrm{CE}$ improved the number of detected peptides from 36 to 87 and the average sequence coverage from $24 \%$ to $38 \%$. Internal calibration was used, and an average mass accuracy of $\mathbf{1 6 . 1} \mathbf{p p m}$ is reported. Finally, diffusion-migration numerical simulations of the iontophoretic fraction collection process have been carried out.

The success of liquid chromatography-mass spectrometry (LC-MS) in biological analysis, and in proteomics in particular, is not only due to its high resolution and reproducibility but also to the relative simplicity for coupling it either to a fraction collector or to a mass spectrometer. As compared to LC, capillary electrophoresis (CE) presents different specificities such as a low or negligible flow rate, a background electrolyte (BGE) that can be highly conductive, and most importantly, the need to maintain an electric field across the capillary during the separation. Because of these features, the development of $\mathrm{CE}-\mathrm{MS}$ has taken much longer than that of LC-MS. ${ }^{1-4}$ However, research on that topic

* To whom correspondence should be addressed. E-mail: hubert.girault@epfl.ch. Fax: +41-21-693-36-67.

(1) Rose, D. J.; Jorgenson, J. W. J. Chromatogr., A 1988, 438, 23-34.

(2) Huang, X.; Zare, R. N. J. Chromatogr. 1990, 516, 185-189.

(3) Huang, X.; Zare, R. N. Anal. Chem. 1990, 62, 443-446.

(4) Keough, T.; Takigiku, R.; Lacey, M. P.; Purdon, M. Anal. Chem. 1992, 64, 1594-1598. is very active, and a number of efficient and robust interfaces are now considered as established technologies and contribute to the development of CE-MS in research fields such as metabolomics, ${ }^{5,6}$ glycoproteomics, ${ }^{7-10}$ or neurochemistry. ${ }^{11}$

In capillary electrophoresis-electrospray ionization mass spectrometry (CE-ESI-MS), the electrospray process can be affected by the nature (conductivity, volatility) of the BGE, and it is important to select a buffer system that does not hinder the MS performances. BGEs of relatively low ionic strength are thus often preferred in order to limit ion suppression. As such choices can affect the separation performances, the hyphenation of $\mathrm{CE}$ to matrix-assisted laser desorption/ionization time-of-flight mass spectrometry (MALDI-TOF MS), which presents a higher tolerance toward salts, represents a valuable alternative. Collection of CE fractions on a MALDI plate also opens the way to on-plate reactions that can be as diverse as protein digestion, ${ }^{12,13}$ chemical modification, ${ }^{14}$ or enrichment of a subclass of analytes. ${ }^{15,16}$

Some commercial CE instruments propose a fraction collection function, where a significant loss of resolution occurs when the electric field is interrupted for changing the outlet vial. Various interfaces have thus been designed to allow fraction collection without current breakdown. Interfaces based on the use of a T-junction allowing the adjunction of a sheath-flow to the $\mathrm{CE}$ effluent have, for example, been reported. ${ }^{8,17,18}$ With such a configuration, MALDI signal intensities are dependent on the amount of analyte deposited and not on its concentration in solution. Still, the area onto which the sample is evaporated has

(5) Monton, M. R. N.; Soga, T. J. Chromatogr., A 2007, 1168, 237-246.

(6) Soga, T. Methods Mol. Biol. (Clifton, NJ) 2007, 358, 129-137.

(7) Amon, S.; Zamfir, A. D.; Rizzi, A. Electrophoresis 2008, 29, 2485-2507.

(8) Amon, S.; Plematl, A.; Rizzi, A. Electrophoresis 2006, 27, 1209-1219.

(9) Bindila, L.; Peter-Katalinic, J.; Zamfir, A. Electrophoresis 2005, 26, 14881499.

(10) Zamfir, A.; Peter-Katalinic, J. Electrophoresis 2004, 25, 1949-1963.

(11) Lapainis, T.; Sweedler, J. V. J. Chromatogr., A 2008, 1184, 144-158.

(12) Vitorino, R.; Guedes, S.; Tomer, K.; Domingues, P.; Duarte, J.; Amado, F. Anal. Biochem. 2008, 380, 128-130.

(13) Li, Y.; Yan, B.; Deng, C.; Tang, J.; Liu, J.; Zhang, X. Proteomics 2007, 7, 3661-3671.

(14) Qiao, L.; Roussel, C.; Wan, J.; Kong, J.; Yang, P.; Girault, H. H.; Liu, B. Angew. Chem., Int. Ed. 2008, 47, 2646-2648.

(15) Qiao, L.; Roussel, C.; Wan, J.; Yang, P.; Girault, H. H.; Liu, B. J. Proteome Res. 2007, 6, 4763-4769.

(16) Bi, H.; Qiao, L.; Busnel, J.-M.; Devaud, V.; Liu, B.; Girault, H. H. Anal. Chem. 2009, 80, 1177-1183.

(17) Lechner, M.; Seifner, A.; Rizzi, A. M. Electrophoresis 2008, 29, 1974-1984.

(18) Muller, O.; Foret, F.; Karger, B. L. Anal. Chem. 1995, 67, 2974-2980. 
to be taken into account. Indeed, in some extreme cases, if large flow rates of sheath-liquid are used together with normal MALDI targets, a loss of sensitivity may occur as the surface area containing the analyte may increase as a function of the deposited volume. To limit this potential drawback, patterned MALDI plates comprising an array of micrometer-sized hydrophilic anchors may be used, as the separated species are then concentrated upon evaporation on a reduced and defined area. ${ }^{19}$ T-junction interfaces employing sheath-liquids are suitable for most CE systems, with or without the presence of an electro-osmotic flow (EOF), but it has been demonstrated that the presence of a makeup liquid flow may potentially induce a suction effect that depends on the sheathflow velocity, capillary dimensions, and general interface geometry. ${ }^{20} \mathrm{~A}$ hydrodynamic flow may then result in the separation capillary leading to a decrease of resolution, due to the presence of a parabolic flow profile.

As a valuable alternative to T-junctions, several liquid junctionbased interfaces have been shown to be effective ${ }^{21-23} \mathrm{~A}$ droplet electrocoupling method, in which CE-separated species are electrokinetically collected into droplets of BGE or matrix previously deposited on a grounded MALDI target, has also been reported several years ago. ${ }^{24}$ Although this technology is suitable for CE system without EOF, current breakdown still occurs at each change of position, thus affecting the overall resolution.

Finally, it has to be noted that electrospray interfaces have also been proposed to continuously deposit CE effluents on a MALDI target. ${ }^{25} \mathrm{~A}$ very high sensitivity has been reached, but this interface presents the same drawbacks as in CE-ESI-MS. Further information on the different hyphenation methods can be found in recently published reviews. ${ }^{26,27}$

In the present work, we introduce a very simple approach that allows the collection of CE-separated species directly on a MALDI target without any current breakdown throughout the separation process. It is mainly based on the use of a capillary, the outer outlet part of which has been coated with a conducting paste. More than 10 years ago, a similar approach was already used for collecting analytes from $\mathrm{CE}{ }^{28}$ This kind of interface was shown to be effective, but its suitability was only demonstrated in the presence of a strong EOF. Here, we show that neutrally coated capillaries generating no significant EOF can also be used. In addition to being compatible with experimental conditions required for the achievement of high-resolution peptide and/or

(19) Johnson, J.; Bergquist, J.; Ekman, R.; Nordhoff, E.; Schurenberg, M.; Kloppel, K. D.; Muller, M.; Lehrach, H.; Gobom, J. Anal. Chem. 2001, 73, 1670-1675.

(20) Mokaddem, M.; Gareil, P.; Belgaied, J. E.; Varenne, A. Electrophoresis 2008, 29, 1957-1964.

(21) Rejtar, T.; Hu, P.; Juhasz, P.; Campbell, J. M.; Vestal, M. L.; Preisler, J.; Karger, B. L. J. Proteome Res. 2002, 1, 171-179.

(22) Preisler, J.; Hu, P.; Rejtar, T.; Moskovets, E.; Karger, B. L. Anal. Chem. 2002, 74, 17-25.

(23) Preisler, J.; Hu, P.; Rejtar, T.; Karger, B. L. Anal. Chem. 2000, 72, 47854795.

(24) Ojima, N.; Shingaki, T.; Yamamoto, T.; Masujima, T. Electrophoresis 2001, 22, 3478-3482.

(25) Wei, H.; Nolkrants, K.; Powell, D. H.; Woods, J. H.; Ko, M. C.; Kennedy, R. T. Rapid Commun. Mass Spectrom. 2004, 18, 1193-1200.

(26) Haselberg, R.; de Jong, G. J.; Somsen, G. W. J. Chromatogr., A 2007, 1159, 81-109.

(27) Huck, C. W.; Bakry, R.; Huber, L. A.; Bonn, G. K. Electrophoresis 2006, 27, 2063-2074.

(28) Chiu, R. W.; Walker, K. L.; Hagen, J. J.; Monnig, C. A.; Wilkins, C. L. Anal. Chem. 1995, 67, 4190-4196. protein separations, one of the main advantages of the present approach certainly stems from its fabrication simplicity. Indeed, no capillary fracture, liquid junction, or sheath-liquid is required.

\section{MATERIALS AND METHODS}

Chemicals. All chemicals used were of analytical reagent grade and obtained from Sigma-Aldrich (Schnelldorf, Switzerland). All buffer and sample solutions were prepared with water produced by an Alpha-Q Millipore system (Zug, Switzerland). Total caseins, $\alpha$-lactalbumin ( $\alpha \mathrm{Lac}), \beta$-lactoglobulin $(\beta \mathrm{Lac})$, bovine serum albumin (BSA), and ribonuclease A (RnaseA) have all been obtained from Sigma-Aldrich (Schnelldorf, Switzerland). Sequencing grade modified trypsin has been obtained from Promega AG (Dübendorf, Switzerland). Human angiotensin III (AngIII), angiotensin II (AngII), and (Sar ${ }^{1}$ )angiotensin I/II(1-7) amide (AngI/II-NH $)_{2}$ have been obtained from Bachem (Bubendorf, Switzerland), and bradykinin (Brad) was from Sigma-Aldrich (Schnelldorf, Switzerland).

Tryptic Digestion. Each protein $\left(0.4-1 \mathrm{mg} \cdot \mathrm{mL}^{-1}\right)$ was dissolved in a $100 \mathrm{mM}$ Tris $/ \mathrm{HCl}(\mathrm{pH}=7.8)$ buffer containing $6 \mathrm{M}$ urea. Dithiothreitol (DTT) was then added to the protein solution at a final concentration of $10 \mathrm{mM}$, and the reduction was allowed to occur for $1 \mathrm{~h}$ at room temperature. Iodoacetamide was added to the reduced protein solution (20 $\mathrm{mM}$ final concentration), and alkylation was performed for $1 \mathrm{~h}$ at room temperature. An excess of DTT was then added to the protein solution to consume the remaining iodoacetamide. After diluting the obtained mixture in water to reduce the concentration of urea to $0.5 \mathrm{M}$, sequencing grade modified trypsin was added to the solution at a protein to enzyme ratio of $50(\mathrm{w} / \mathrm{w})$. The digestion was then allowed to occur overnight at $37^{\circ} \mathrm{C}$. Peptide mixtures were then lyophilized and further reconstituted in water if a preconcentration step was required. The obtained protein tryptic digests were then analyzed directly by $\mathrm{CE}$ or MS without any further purification step.

Capillary Electrophoresis. The CE experiments were carried out with an $\mathrm{HP}^{3 \mathrm{D}} \mathrm{CE}$ apparatus (Agilent, Waldbronn, Germany) equipped with a diode-array detector, an autosampler, and a power supply able to deliver up to $30 \mathrm{kV}$. Fused-silica capillaries were obtained from BGB Analytik AG (Böckten, Switzerland) and coated with hydroxypropylcellulose (HPC) in the laboratory following the procedure described earlier by Shen and Smith. $^{29}$ Solutions of $10 \%$ acetic acid and ammonium acetate ( $\mathrm{pH}=4,250 \mathrm{mM}$ ionic strength) have been employed in $\mathrm{CE}$ as BGE and leading electrolyte (LE), respectively. Injection volumes have been calculated using the Poiseuille equation where the fluid viscosity was approximated to the one of water, at $20{ }^{\circ} \mathrm{C}(0.89 \mathrm{cP})$.

CE-MALDI Interface. One extremity of the neutrally coated capillary was painted with a silver ink from Ercon (Wareham, MA) over a length of about $8 \mathrm{~cm}$. Then, the ink was cured at $80^{\circ} \mathrm{C}$ for at least $90 \mathrm{~min}$ in an oven. The capillary was placed in a $\mathrm{CE}-\mathrm{MS}$ cassette and then positioned in the $\mathrm{CE}$ apparatus, the nonpainted extremity being in the apparatus and the painted one placed in a ceramic holder, being an integrated part of a homemade robotic system able to move in all three axes and computer-controlled via a Labview program (National Instruments, Austin, TX). More precisely,

(29) Shen, Y.; Smith, R. D. J. Microcolumn Sep. 2000, 12, 135-141. 


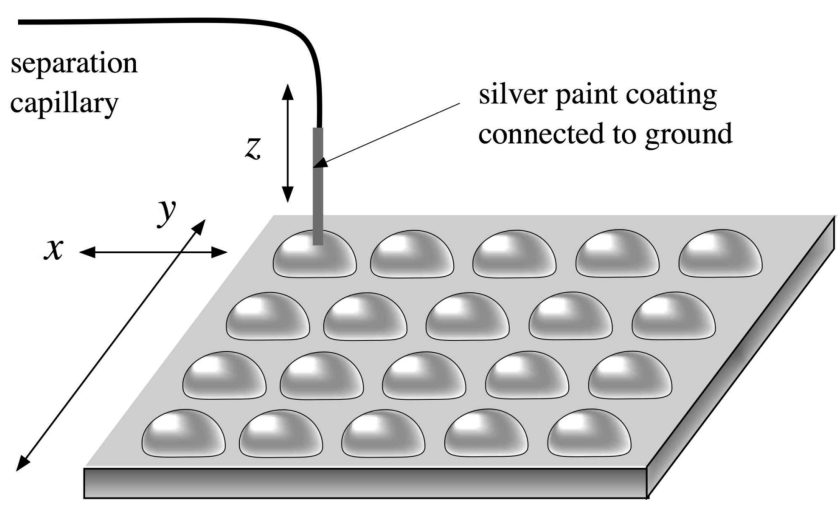

Figure 1. Schematic of the robotized fraction collection interface including a MALDI plate mounted on an $x, y$-stage and a separation capillary silver-coated at the tip for a connection to ground and mounted on a $z$-axis holder.

a MALDI target is placed on the stage of the robotic system, which can move in the $x, y$-plane, and movement in the $z$-direction is accomplished by moving the outlet part of the capillary. The schematic of the CE-MALDI interface is represented in Figure 1. Whatever the support used for fraction collection, the painted extremity of the capillary is grounded and is dipped in a small volume of receiving solution to deliver the CE-separated species. First, the painted extremity of the capillary is dipped in a BGE for a certain time, and then the fraction collection is achieved repeatedly by lifting the capillary and dipping it at the next position.

MALDI-TOF MS. When the samples were directly collected on a MALDI target, $0.75 \mu \mathrm{L}$ of a rather diluted $\alpha$-cyano-4hydroxycinnamic acid (CHCA) matrix solution $\left(2 \mathrm{mg} \cdot \mathrm{mL}^{-1}\right.$ CHCA in $0.1 \%$ trifluoroacetic acid/acetonitrile (TFA/ACN) (50/ 50)) were first deposited and subsequently allowed to dry on each position of an AnchorChip MALDI target (Bruker, Bremen, Germany). Depending on the requirements, 4 or 10 $\mu \mathrm{L}$ of $10 \%$ acetic acid was then deposited before the separation step on each position. After collection, the droplets were allowed to dry at room temperature under atmospheric pressure. Then, $0.5 \mu \mathrm{L}$ of $0.1 \%$ TFA/ACN (50/50) was deposited on each position for recrystallizing the peptides within the matrix. This last recrystallization step was found to be mandatory as its avoidance led to very poor spectra or even the absence of signals, this certainly being a result of the poor solubility of CHCA matrix in aqueous media. Under these conditions, the presence of a high content of acetic acid in the collection droplet did not appear as a deleterious parameter for the cocrystallization of the analyte within the matrix. For the direct analysis using the AnchorChip target, the same previously described thin-layer spotting method was used.

For the different spotting conditions, an internal calibration was performed with matrix solutions containing the following peptides: angiotensin I (1296.685 Da) and II (1046.542 Da), substance P (1347.735 Da), bombesin (1619.822), and ACTH_Clip (18-39) (2465.198). All MALDI-TOF experiments have been carried out on a Bruker (Bremen, Germany) Microflex equipped with a nitrogen laser operating at $337 \mathrm{~nm}$. Positively charged ions were then detected, and the sum of 400 single spectra was used for data treatment. All MS results are summarized in different tables available as Supporting Information. Due to instrument limitations, peptide identification has only been performed with $\mathrm{MS}^{1}$ data. Mass accuracy was limited to $50 \mathrm{ppm}$, and in the case where several peptides, originating from several proteins, satisfied this condition, preference were given to fully alkylated species. If such criteria could not be applied, isobaric species were simply excluded from the data treatment.

Numerical Model for Diffusion-Migration Simulations. A detailed description of the numerical model is given in the Supporting Information.

\section{RESULTS AND DISCUSSION}

Impact of Spotting on Separation Resolution and Efficiency. As a first step, we have studied with UV detection the evolution of the resolution as a function of the spotting

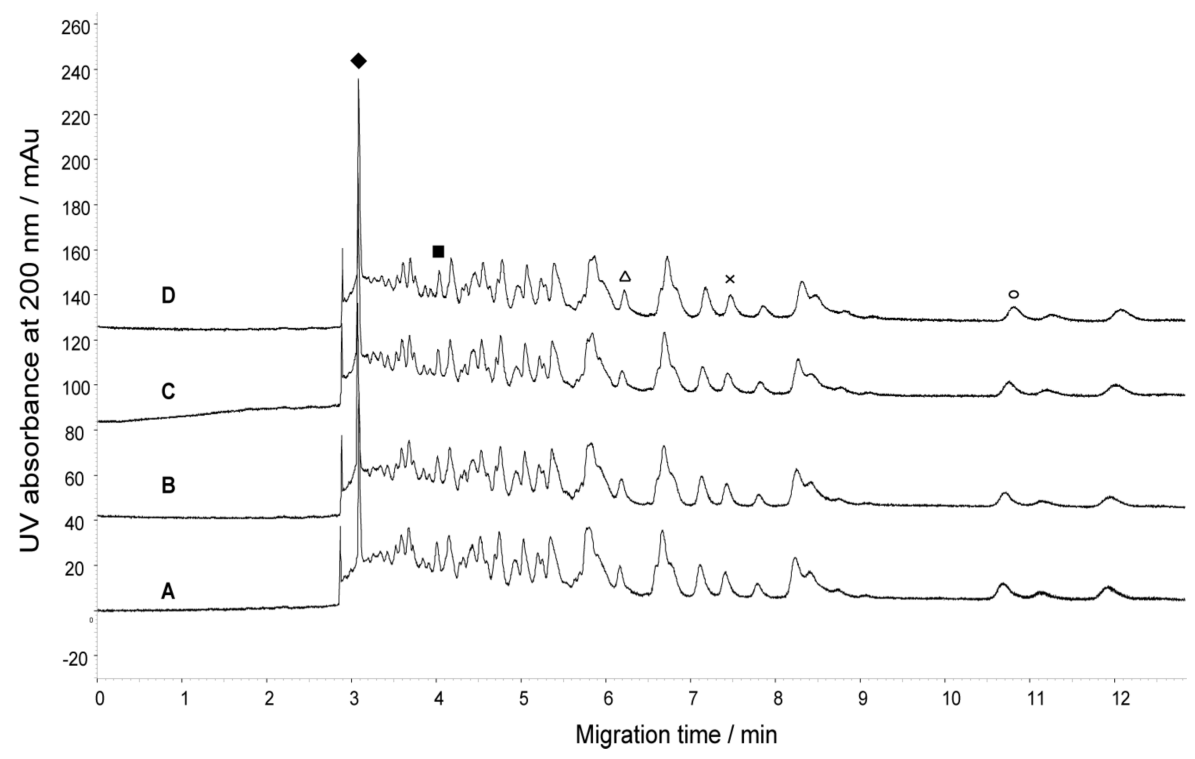

Figure 2. Impact of spotting interval time on separation resolution. Experimental conditions: HPC-coated capillary, total/effective length 80/ $21.5 \mathrm{~cm} \times 50 \mu \mathrm{m}$ i.d.; voltage, $30 \mathrm{kV}$; current, $12 \mu \mathrm{A}$; UV absorbance at $200 \mathrm{~nm}$; BGE, $10 \%$ acetic acid; sample, tryptic digest of $\alpha$-lactalbumin $(35 \mu \mathrm{M})$ and $\beta$-lactoglobulin $(27 \mu \mathrm{M})$; sample injection, 30 mbar $30 \mathrm{~s}$. (A) No spotting. (B) Spotting every $60 \mathrm{~s}$. (C) Spotting every $30 \mathrm{~s}$. (D) Spotting every $15 \mathrm{~s}$. Labeled peaks have been chosen for constructing Supporting Information Table S-1. 

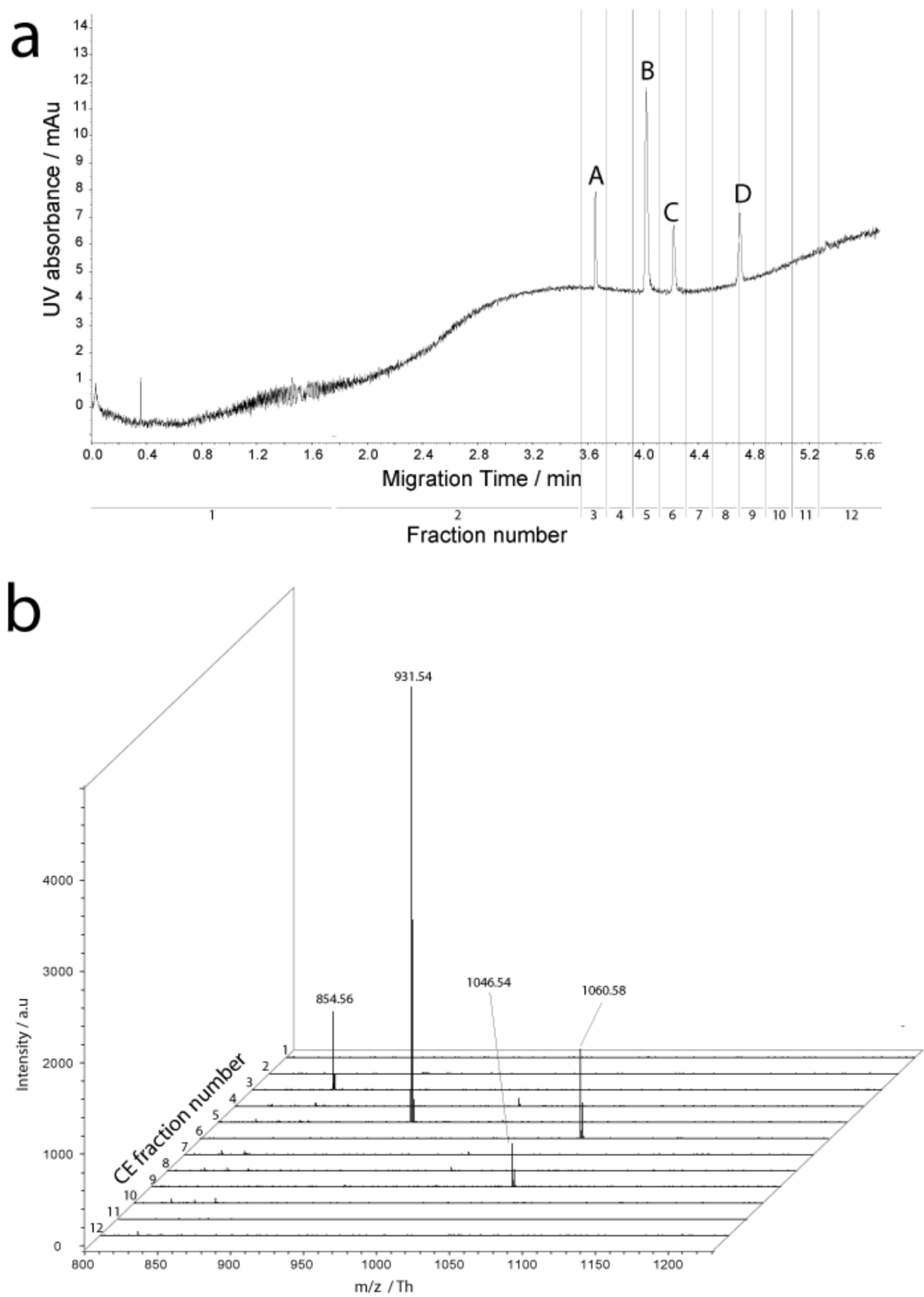

Figure 3. CE-UV and CE-MALDI-TOF MS of a four-peptide sample mixture. Experimental conditions: (a) HPC-coated capillary, total/effective length 50/41.5 cm $\times 50 \mu \mathrm{m}$ i.d.; BGE, $10 \%$ acetic acid; voltage, $30 \mathrm{kV}$; current, $20 \mu \mathrm{A}$; UV absorbance at $200 \mathrm{~nm}$; sample, Ang I/II-NH 2 (16 $\mu \mathrm{M})$, Ang III $(22 \mu \mathrm{M})$, Brad $(13 \mu \mathrm{M})$, and Ang II $(13 \mu \mathrm{M})$; sample injection, 30 mbar $2.5 \mathrm{~s}$. (b) HPC-coated capillary, total length $41.5 \mathrm{~cm} \times 50 \mu \mathrm{m}$ i.d.; BGE, 10\% acetic acid; voltage, $24.9 \mathrm{kV}$; current, $20 \mu \mathrm{A}$; sample, Ang I/II- $\mathrm{NH}_{2}(16 \mu \mathrm{M})$, Ang III $(22 \mu \mathrm{M})$, Brad (13 $\left.\mu \mathrm{M}\right)$, and Ang II (13 $\left.\mu \mathrm{M}\right)$; sample injection, 30 mbar $2 \mathrm{~s}$. Collection on the MALDI plate: $4 \mu \mathrm{L}$ of BGE on each collection position. Fraction collection durations: $105 \mathrm{~s}$ for the two first fractions, then $10 \mathrm{~s}$ per fraction. See the Materials and Methods section for MS experimental conditions.

time interval ranging from 15 to $60 \mathrm{~s}$ when using a negligible EOF. It is an important parameter to determine whether the developed system is suited to CE collection. A neutrally coated capillary has been used together with a rather complex test 


\section{Table 1. Number of Identified Peptides and Sequence Coverage if Using Direct MALDI-TOF MS or CE-MALDI-TOF MS for the Analysis of the Peptide Test Mixturea}

\begin{tabular}{lccccc} 
& \multicolumn{2}{c}{ CE-MALDI-TOF MS } & & direct MALDI-TOF MS \\
\cline { 2 - 3 } & $\begin{array}{c}\text { no. of } \\
\text { identified } \\
\text { peptides }\end{array}$ & $\begin{array}{c}\text { sequence } \\
\text { coverage (\%) }\end{array}$ & & $\begin{array}{c}\text { no. of } \\
\text { identified } \\
\text { peptides }\end{array}$ & $\begin{array}{c}\text { sequence } \\
\text { coverage (\%) }\end{array}$ \\
$\alpha$ s1-casein & $\mathbf{6}$ & $\mathbf{3 3}$ & & 3 & 17 \\
$\alpha$ S2-casein & $\mathbf{6}$ & $\mathbf{1 8}$ & & 0 & 0 \\
$\beta$-casein & 1 & 8 & & 1 & 8 \\
$\kappa$-casein & $\mathbf{2}$ & $\mathbf{1 1}$ & & 1 & 5 \\
$\alpha$-lactalbumin & $\mathbf{7}$ & $\mathbf{4 5}$ & & 6 & 32 \\
$\beta$-lactoglobulin & $\mathbf{8}$ & 55 & & 6 & $\mathbf{5 9}$ \\
RNA & $\mathbf{7}$ & $\mathbf{5 8}$ & & 3 & 40 \\
BSA & $\mathbf{5 0}$ & $\mathbf{7 9}$ & & 16 & 34
\end{tabular}

total no. of av sequence total no. of av sequence identified peptides coverage (\%) identified peptides coverage (\%)
87
38.4
36
24.4

${ }^{a}$ Experimental conditions for CE: HPC-coated capillary, total/ effective length $80 / 21.5 \mathrm{~cm} \times 50 \mu$ m i.d.; voltage, $30 \mathrm{kV}$; current, 12 $\mu \mathrm{A} ; \mathrm{BGE}, 10 \%$ acetic acid; sample, tryptic digest of total caseins (14 $\mu \mathrm{M}), \alpha$-lactalbumin $(11 \mu \mathrm{M}), \beta$-lactoglobulin $(9 \mu \mathrm{M})$, bovine serum albumin $(3 \mu \mathrm{M})$, ribonuclease A $(15 \mu \mathrm{M})$; sample injection, 30 mbar $200 \mathrm{~s}$. Leading electrolyte (ammonium acetate $\mathrm{pH} 4,250 \mathrm{mM}$ ionic strength) injection prior to sample injection: $30 \mathrm{mbar} 40 \mathrm{~s}$. See the Materials and Methods section for MS experimental conditions.

sample mixture, containing the tryptic digests of $\alpha \mathrm{Lac}$ and $\beta$ Lac. The results of the capillary zone electrophoresis separation are shown in Figure 2.

As a control experiment, a separation without any fraction collection has also been achieved. Here, the detector is located at only $21.5 \mathrm{~cm}$ from the inlet when the total length of the capillary is $80 \mathrm{~cm}$. This results in a rather poor resolution as the length of the injected sample zone accounts for $5 \%$ of the reported effective length. Still, the results of Figure 2 show that the spotting process has basically no impact on the in-capillary resolution. In this experiment, the robot needs $1.8 \mathrm{~s}$ to reposition the capillary outlet from one position to the next. Considering that $0,13,26$, and 52 fractions were collected, it is clear that the electromigration never stops during one analysis. This is confirmed by the fact that no current breakdown takes place throughout the separation process, whatever the collection time. A thin film of liquid covers the outlet end of the capillary ensuring a wetting of the electrode during its displacement, its volume being large enough for allowing it to act as a buffer reservoir during the required time.

To confirm quantitatively that the spotting process has no significant influence on the in-capillary resolution, we provide in Supporting Information Table S-1 the peak efficiency of several peaks as a function of the number of collected fractions. Five peaks, covering the full separation window, have been chosen as marked on trace D of Figure 2.

Under the present experimental conditions, as many fractions as required can be collected without any detrimental effect on the separation process, and thus the spotting interval can be optimized as a function of the sample complexity to provide the highest identification rate.

Direct Spotting on a MALDI Plate. To demonstrate the iontophoretic fraction collection approach, a test mixture consist- ing of four peptides has first been considered. It has been analyzed both by CE-UV (Figure 3a) and CE-MALDI-TOF MS (Figure $3 b)$. These experiments have been performed under a rather high electric field $(600 \mathrm{~V} / \mathrm{cm})$ while minute peptide quantities (from 32 to $55 \mathrm{fmol}$ ) have been injected into the $\mathrm{CE}$ capillaries. As fast migrating peptides had to be collected, a short fraction collection time of $10 \mathrm{~s}$ has been used. Considering that each peptide zone was spread over a time ranging from less than $2 \mathrm{~s}$ to about $5 \mathrm{~s}$ (Figure 3a) and that the MS spectra of each collected fraction showed the presence of a maximum of one species, it appears that the interface does not induce any significant carryover that would hinder both the sensitivity and the resolution of the experiments.

To further characterize the fraction collection interface, 130 $\mathrm{nL}$ of a more complex sample mixture containing the tryptic digests of caseins, $\alpha$ Lac, $\beta$ Lac, RnaseA, and BSA has been analyzed. Amounts of protein tryptic digests ranging from less than $400 \mathrm{fmol}$ (BSA) to $1.9 \mathrm{pmol}$ (RnaseA) were thus injected. Prior to the sample injection, a short zone $(\approx 25 \mathrm{~nL})$ of ammonium

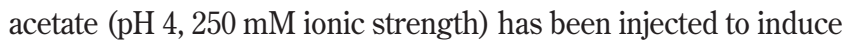
a transient isotachophoresis (t-ITP) preconcentration step, the expected effect being a concomitant increase of efficiency and resolution. ${ }^{30}$

Long spotting intervals have been chosen at the beginning of the separation when no peptide reaches yet the end of the capillary. Spotting intervals have then been gradually diminished down to $20 \mathrm{~s}$ and finally increased back to $60 \mathrm{~s}$ for the collection of the slow migrating peptides. In total, 72 fractions have been collected. To assess the benefits linked to the hyphenation of CE with MALDI, we have performed in parallel a direct MALDI MS of the nonseparated sample mixture (materials available as Supporting Information). The same prestructured MALDI plate has been used, and the same thin-layer deposition technique has been applied. Different dilutions of the sample mixture have been used, and the best results have been obtained with protein tryptic digest amounts ranging from $230 \mathrm{fmol}$ (BSA) to $1.2 \mathrm{pmol}$ (RnaseA) (corresponding spectrum available as Supporting Information). It is worth to precise that these amounts are of the same magnitude as those injected in CE. Table 1 compares, for each protein, the number of identified peptides and the respective sequence coverage, both for direct MALDI-TOF MS and CE-MALDITOF MS. Whereas the direct MALDI MS analysis yields an identification of 36 peptides, 87 were identified with the integration of a CE separation step. As a result, a significant improvement of the average sequence coverage is reported, thereby demonstrating the usefulness of integrating a separation step prior to MS analysis.

To further characterize the present CE-MALDI interface, we have carefully analyzed the MS data to assess the analyte carryover from fraction to fraction. Results are shown in Figure 4. A few peptides are spread over four or five neighboring fractions, but most of them are present in only one or two fractions. This shows that there is no significant oversampling from the $\mathrm{CE}$ separation to the MALDI plate. Most importantly, it shows that there is no significant carryover, its extent being not expected to compromise the subsequent MALDI analysis. These results indicate that each peptide exiting from the capillary diffuses efficiently into the collection droplet.

(30) Busnel, J. M.; Lion, N.; Girault, H. H. Electrophoresis 2008, 29, 1565-1572. 


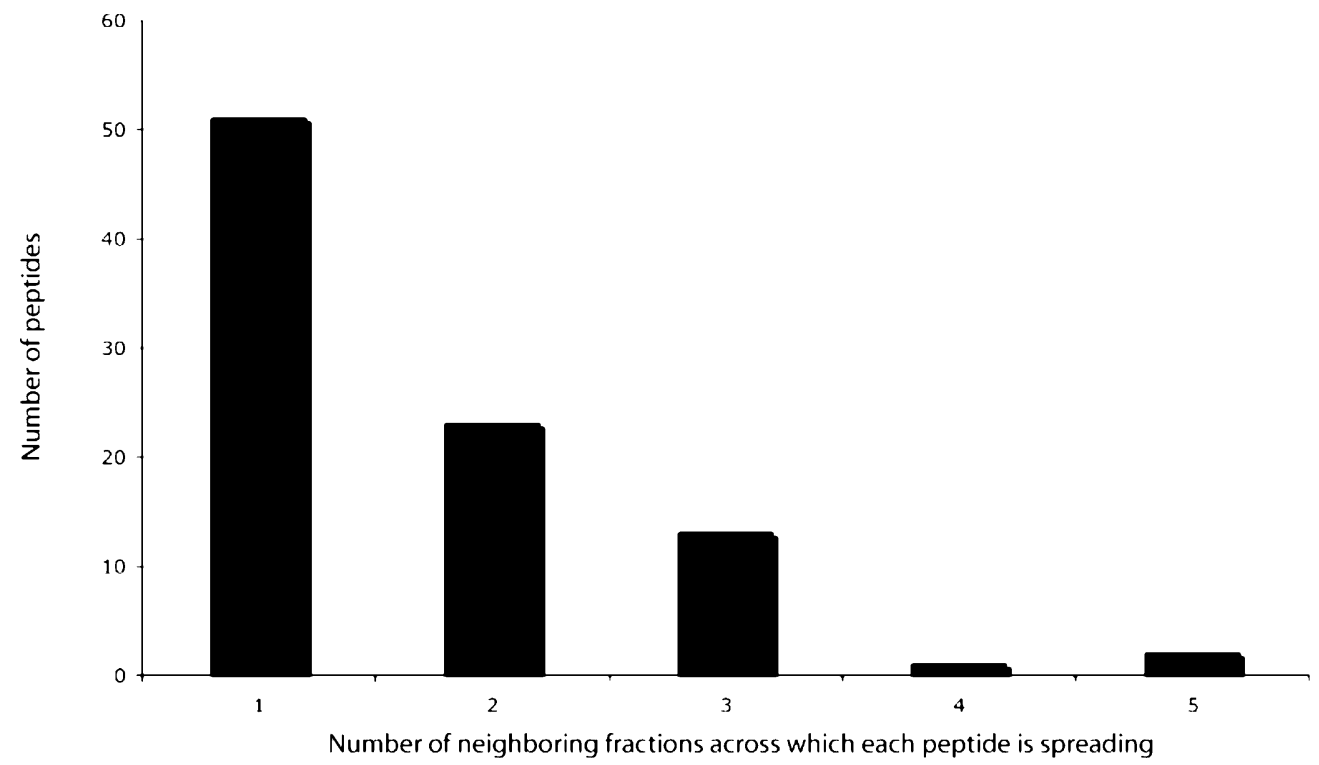

Figure 4. Spreading of the peptides across neighboring fractions. Experimental conditions: same as in Table 1.

Under its present form, the need for a large receiving droplet volume certainly constitutes the main limiting factor of the proposed method. Indeed, although the present strategy certainly provides a simplification of the experimental setup as compared to interfaces based on the use of a sheath-liquid or a liquid junction, it requires the use of prestructured MALDI targets. Indeed, if such a patterned plate is not employed, lower collection volumes have to be used, thereby precluding long analysis times and certainly inducing a significant loss of sensitivity, as the sample would not be, upon evaporation, concentrated on a reduced surface area.

Simulation of the Iontophoretic Spotting Process. To improve the understanding of the reported iontophoretic spotting process, diffusion-migration simulations have been carried out. The spotting process has been simulated from the moment where a $1 \mathrm{~mm}$ sample plug arrives at the outlet of the capillary and further performed to simulate a total collection time of $40 \mathrm{~s}$. A peptide presenting average values of electrophoretic mobility and diffusion coefficient has been chosen for the calculation. (See the Supporting Information for a detailed description of the simulation part.)

Considering the results of this simulation part, it can be concluded that the coating of the outer outlet part of the separation capillary has to be properly optimized as it can influence a lot the obtained results. Especially, in the case of electrophoretic separations carried out under high electric field or under conditions that induce large electrophoretic mobilities of the separated analytes, the outer coating of the capillary outlet should ensure the lowest film thickness while allowing current application when the capillary is moved from fraction to fraction.

\section{CONCLUSIONS}

An iontophoretic approach for collecting migrating analytes from $\mathrm{CE}$ has been developed. Depending on the fractionation requirements, CE-separated species can be collected either directly on a MALDI target or in a multiwell plate. This setup is based on the painting of the outer outlet part of the separation capillary with a silver conducting paste. The efficiency of the developed interface does not rely on the use of a sheath-flow or a liquid junction, and neither hydrodynamic nor electro-osmotic flow is required. Neutrally coated capillaries, producing negligible EOF, can thus be used for the achievement of high-resolution peptide or protein separations. As the fraction collection process does not hinder the resolution of the CE separation, as many fractions as desirable can be collected to meet the requirements imposed by the complexity of the studied sample. No significant carryover of peptides from fraction to fraction has been detected. As compared to the direct MALDI analysis of the sample studied where 36 peptides have been identified, the integration of $\mathrm{CE}$ separation combined to the use of the developed fraction collection device allowed the identification of 87 peptides with an average mass accuracy of $16.1 \mathrm{ppm}$. Moreover, as a final step in this study, the developed interface has been modeled and the iontophoretic process simulated. These simulations highlight the key parameters to optimize if electrophoretic separations under very high electric field have to be achieved. As the present methodology is cheap, highly efficient, and easy to integrate, the proposed CE-MALDI interface certainly represents a practical solution to hyphenate $\mathrm{CE}$ to MALDI MS. Still, the need to use large volume collection droplets so far constitutes a drawback, and prestructured MALDI targets have to be employed to concentrate the samples on a small surface area.

\section{SUPPORTING INFORMATION AVAILABLE}

Additional information as noted in text. This material is available free of charge via the Internet at http://pubs.acs.org.

Received for review October 28, 2008. Accepted April 3, 2009.

AC900128Q 